\title{
INDEX for Volume 95
}

M. Bailey, Local classification of generalized complex structures. .

D. Baskin \& J. Wunsch, Resolvent estimates and local decay of

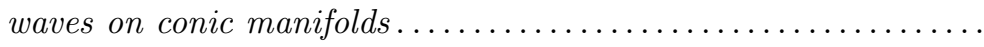

K.-J. Böröczky, Stronger versions of the Orlicz-Petty projection

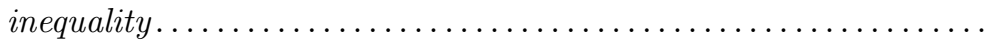

I. Chatterji, Y. de Cornulier, G. Mislin, \& Ch. Pittet, Bounded

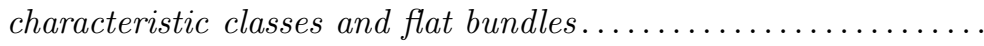

T.H. Colding, T. Ilmanen, W.P. Minicozzi II, \& B. White, The round sphere minimizes entropy among closed self-shrinkers.....

C. De Lellis \& D. Tasnady, The existence of embedded minimal

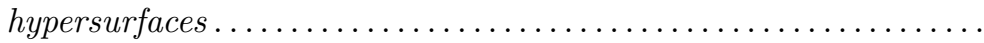

M. Eichmair, G. J. Galloway, \& D. Pollack, Topological censorship

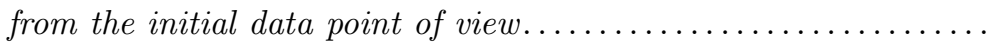

L. Funar \& J. Marché, The first Johnson subgroups act ergodically

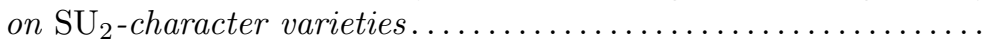

L.-H. Huang \& D. Wu, Hypersurfaces with nonnegative scalar curvature

N.C. Leung, X. Wang, \& K. Zhu, Thin instantons in $G_{2}$-manifolds and Seiberg-Witten invariants ......................

S.-Y. Li \& X. Wang, An Obata-type theorem in CR geometry.... 419

M. McQuillan \& D. Panazzolo, Almost étale resolution of foliations

S.-H. Min, Embeddedness of proper minimal submanifolds in ho-

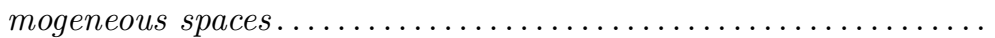

W. Müller \& J. Pfaff, Analytic torsion and $L^{2}$-torsion of compact

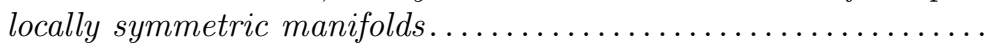

H. Rosenberg, F. Schulze, \& J. Spruck, The half-space property and entire positive minimal graphs in $M \times \mathbb{R} \ldots \ldots \ldots \ldots \ldots$

Y.-C. Tu, S. S.-T. Yau, \& H. Zuo, Nonconstant CR morphisms between compact strongly pseudoconvex $C R$ manifolds and étale covering between resolutions of isolated singularities...........

C. Wendl, Non-exact symplectic cobordisms between contact 3-

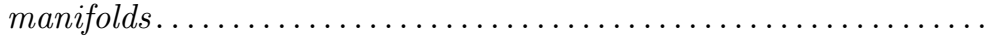

